\title{
How Do Molecular Motions Affect Structures and Properties at Molecule and Aggregate Levels?
}

Deshuang Tu, ${ }^{[a] \# ~ J i a n y u ~ Z h a n g, ~}{ }^{[a] \#}$ Yunxiao Zhang, ${ }^{[a]}$ Herman H. Y. Sung, ${ }^{[a]}$ Lijie Liu, ${ }^{[a]}$ Ryan T. K. Kwok, ${ }^{[a]}$ Jacky W. Y. Lam, ${ }^{*[a]}$ Ian D. Williams, ${ }^{[a]}$ Hong Yan ${ }^{*[b]}$ and Ben Zhong Tang ${ }^{*[a, c, ~ d, ~ e] ~}$

\section{Affiliations:}

${ }^{a}$ Department of Chemistry, Hong Kong Branch of Chinese National Engineering Research Center for Tissue Restoration and Reconstruction and Institute for Advanced Study, The Hong Kong University of Science and Technology, Clear Water Bay, Kowloon, Hong Kong 999077, China

${ }^{b}$ State Key Laboratory of Coordination Chemistry, Jiangsu Key Laboratory of Advanced Organic Materials, School of Chemistry and Chemical Engineering, Nanjing University, Nanjing 210023, China

${ }^{\mathrm{c}}$ Center for Aggregation-Induced Emission, State Key Laboratory of Luminescent Materials and Devices, SCUT-HKUST Joint Research Institute, South China University of Technology

Tianhe Qu, Guangzhou 510640, China

${ }^{\mathrm{d}}$ Guangdong-Hong Kong-Macao Joint Laboratory of Optoelectronic and Magnetic Functional Materials

eShenzhen Institute of Molecular Aggregate Science and Engineering, School of Science and Engineering, The Chinese University of Hong Kong, Shenzhen, 2001 Longxiang Boulevard, Longgang District, Shenzhen City, Guangdong 518172, China

${ }^{\#}$ D. T. and J. Z. contributed equally to this work

Correspondence and requests for materials should be addressed to J. L. (E-mail: chjacky@ust.hk) or to H. Y. (E-mail: hyan1965@nju.edu.cn) or to B. Z. T. (E-mail: tangbenz@,cuhk.edu.cn) 


\begin{abstract}
Molecular motions are essential natures of matters, and play important roles in their structures and properties. However, owing to the diversity and complexity of structures and behaviors, the study of motion-structure-property relationship remains a challenge, especially at all levels of structural hierarchy from molecule to macro-object. Herein, luminogens showing aggregation-induced emission (AIE), namely 9-(pyrimidin-2-yl)-carbazole (PyCz) and 9-(5-R-pyrimidin-2-yl)-carbazole; $\mathrm{R}=\mathrm{Cl}(\mathrm{ClPyCz}), \mathrm{Br}(\mathrm{BrPyCz})$ and $\mathrm{CN}(\mathrm{CyPyCz})$ were designed and synthesized, to decipher the dependence of materials' structures and properties on molecular motions at molecule and aggregate levels. Experimental and theoretical analysis demonstrated that the active intramolecular motions in the excited state of all molecules at single molecule level imparted them with more twisted structural conformations and weak emission. However, owing to the restriction of intramolecular motions in the nano/macro aggregate state, all the molecules assumed less twisted conformations with bright emission. Unexpectedly, intermolecular motions could be activated in the macro crystals of $\mathrm{ClPyCz}, \mathrm{BrPyCz}$ and $\mathrm{CyPyCz}$ through the introduction of external perturbations, and synergic strong and weak intermolecular interactions allowed their crystals to undergo reversible deformation, which effectively solved the problem of the brittles of organic crystals, meanwhile imparted them with excellent elastic performance. Thus, the present study provided insights on the motionstructure-property relationship at each level of structural hierarchy, and offered a paradigm to rationally design multifunctional AIE-based materials.
\end{abstract}




\section{Introduction}

The world is full of materials, and motion is the mode of existence of matters. As one of the basic natures, motion always influences the structures and properties of matters. In modern science, matters are constructed from microcosmic atoms, then molecules, and finally to macroscopic objects, from which theories of structural hierarchy are built. Besides, the inherent properties of matters are closely related with structures. Therefore, clarifying the relationship between motion, structure and property (MSP) at each level of structural hierarchy not only provides insights on fundamental science but also paves the way for practical applications.

At single molecule level, dynamic intramolecular motion is dominant through rotation of covalent bond and vibration of skeleton, and influences the structural conformation. Molecules with free molecular motion are usually sensitive to surrounding environment and thus display properties such as twisted intramolecular charge transfer (TICT) ${ }^{1-2}$. In contrast to molecule level, the MSP relationship in the aggregate state is bound up with many factors. Except restricted intramolecular motion, molecules may undergo multiple intermolecular interactions, different motional modes, and express structural and behavioral diversities at each level of structural hierarchy. Except these internal effects, external stimuli such as light irradiation, magnetic field and mechanical force are capable of influencing molecular motion and property. ${ }^{3-5}$ Consequently, all these mutual factors give rise to complicated MSP relationship at the aggregate level. On the other hand, the direct observation of molecular motions is not easy because of untouchable change of motion in isolated molecule. ${ }^{6}$ Although molecular motions can be characterized using nuclear magnetic resonance, electron microscopes, spectroscopies, etc., ${ }^{7-11}$ these technologies cannot perfectly present aggregate-state molecular motions in real time and in-situ manner. Therefore, MSP study at all levels of structural hierarchy is still attractive but challenging.

Aggregation-induced emission (AIE) is an unusual photophysical phenomenon in which aggregates exhibit brighter emission than single molecules in the solution state. ${ }^{12} \mathrm{After}$ twenty-year effort, AIE research has made great advances from photophysical phenomenon to materials, mechanistic study and high-tech applications. ${ }^{13-21}$ In particular, it demonstrates that molecular motion plays a key role in photophysical processes. Namely, the free molecular motion of AIE luminogens (AIEgens) in the solution state dissipates excited-state energy through the non-radiative relaxation channel to result in quenched or weak emission in the solution state. ${ }^{22}$ However, when the molecular conformation is rigidified through the restriction of intramolecular motions in the aggregate state. AIEgens will show intense emission. ${ }^{23}$ In this regard, AIEgens provide an excellent platform to explore MSP relationship. On the other hand, recent AIE research was been focused on studying photophysical behaviors. However, exploration of other physical or chemical properties such as elasticity is virtually unexplored. ${ }^{13-19}$ In addition, aggregation performs like a double-edged sword that promote bright emission but deactivates some other properties like elasticity in the crystalline phase (Figures S1 and S2, in the Electronic Supporting Information). ${ }^{24}$ Without doubt, realization of AIE-active multifunctional organic crystals is fantastic for their potential applications in material science and biological applications. 
To investigate MSP relationship at all levels of structural hierarchy and realize additional properties using AIEgens, the following requirements on the molecular design should be followed: (i) flexible molecular structure to enable the molecules to undergo large molecular motion in the isolated state; (ii) structural conformation-dependent properties at each level of structural hierarchy, especially in macro aggregates; and (iii) synergistic strong and weak intermolecular interactions in macro-crystal to afford multiple intermolecular motions to realize diversified properties. Except these requirements, it will be beneficial to the study the MSP relationship if the AIEgens have good crystallinity and simple molecular structures. Based on these considerations, AIEgens with TICT effect were considered in this work. Through optimized molecular design and subtle crystal engineering, electron-donating carbazole group and electron-deficient molecular rotor of pyrimidinyl unit were chosen to synthesize several simple AIEgens. Interestingly, these easyaccessed AIEgens were capable of elaborating the MSP relationships at all structural hierarchies from single molecule to nano- and macro aggregate levels, and their crystals displayed unexpected elastic behavior. Thus, the present study not only shed light on the MSP relationship, but also presented a facile strategy to construct AIE-active elastic organic crystals with high performance.

\section{Results and discussion}

\section{Molecular design and synthesis}

To obtain motional molecules to explore the MSP relationship, carbazole, which is a simple chromophore with electron-donating property, was selected to construct AIEgens with different molecular rotors. On the other hand, phenyl group is a classical molecular rotor, and was first considered and introduced to generate 9-phenylcarbazole $(\mathrm{PhCz})$. The calculated potential energy surface (PES) revealed that $\mathrm{PhCz}$ possessed a twisted conformation in both ground $\left(\mathrm{S}_{0}\right)$ and excited $\left(\mathrm{S}_{1}\right)$ states due to the large steric hinderance between two $\mathrm{C}-\mathrm{H}$ units of phenyl ring and carbazolyl group (Figure 1a). Thus, PhCz fails to satisfy the purpose of visualization of structural conformation and property changes at different levels of structural hierarchy. Therefore, pyrimidinyl unit, of which the two $\mathrm{C}-\mathrm{H}$ units of phenyl ring were replaced by nitrogen atoms, was utilized as an electrondeficient rotor to build 9-(pyrimidin-2-yl)-carbazole (PyCz). Results from theoretical calculation indicated that $\mathrm{PyCz}$ showed a planar conformation in $\mathrm{S}_{0}$ but adopted a twisted conformation in $\mathrm{S}_{1}$ due to the molecular motion (Figure 1b). Therefore, according to our previous studies, ${ }^{12,14,25}$ such an intramolecular motion could be blocked in the aggregate state to endow the molecule with AIE activity. Meanwhile, $\mathrm{Cl}$ substituent was further introduced at the para position of the pyrimidinyl unit to give 9-(5-chloropyrimidin-2-yl)-carbazole (ClPyCz) showing multiple intermolecular interactions in the crystalline state. As suggested by the calculated PES (Figure 1b), CIPyCz also displayed flexible molecular motion and TICT effect in the isolated state.

On the basis of these considerations, two target compounds, namely $\mathrm{PyCz}$ and $\mathrm{ClPyCz}$, were synthesized via an effective $\mathrm{C}-\mathrm{N}$ coupling reaction (Schemes $\mathrm{S} 1$ and $\mathrm{S} 2$ ), and carefully characterized by NMR, Mass spectroscopies. Moreover, crystals of two compounds were obtained via simple solvent evaporation process and their structures were determined (Figure S3 and Table $\mathrm{S} 1)$.

\section{MSP study at molecule and nano aggregate levels}


Photoluminescence (PL) spectra of $\mathrm{PyCz}$ and $\mathrm{ClPyCz}$ were first obtained (Figure S4 and S5) to explore MSP at the molecule level. Both of them showed a broad emission peak from 400 to 600 $\mathrm{nm}$ with obvious solvent chromic properties. Besides, Lippert-Mataga equation also revealed the relatively large slopes ( $>8000 \mathrm{~cm}^{-1}$, Figures S6 and S7) to illuminate the TICT property of the two compounds. ${ }^{26}$ In contrast, $\mathrm{PhCz}$ without donor-acceptor structure showed the emission of carbazole with peak at around 345 and $360 \mathrm{~nm}$ (Figure S8). These data verified the free intramolecular motion of $\mathrm{PyCz}$ and $\mathrm{ClPyCz}$ in the solution state, which provided an effective channel in the excited state to access the twisted molecular conformation with TICT property.
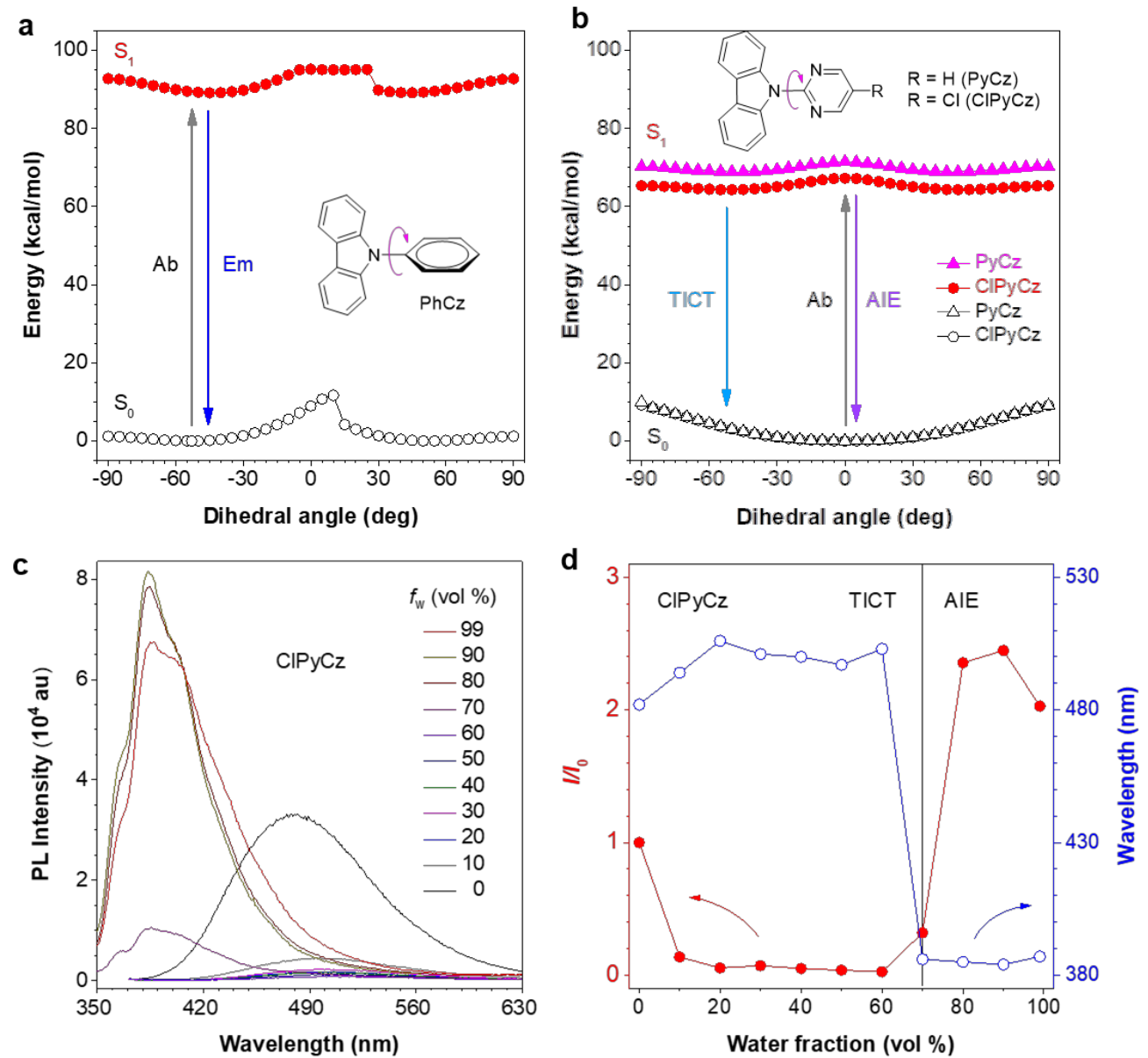

Figure 1. Potential energy surfaces for (a) $\mathrm{PhCz}$ and (b) $\mathrm{ClPyCz}$ and $\mathrm{PyCz}$ with different dihedral angles in the ground state $\left(\mathrm{S}_{0}\right)$ and excited state $\left(\mathrm{S}_{1}\right) . \mathrm{Ab}=$ absorption, $\mathrm{Em}=$ emission, $\mathrm{TICT}=$ twisted intramolecular charge transfer, AIE = aggregation-induced emission. (c) Photoluminescence (PL) spectra of ClPyCz in acetonitrile/water mixtures with different water fractions $\left(f_{\mathrm{w}}\right)$. (d) Plot of relative PL intensity $\left(I / I_{0}\right)$ of $\mathrm{ClPyCz}$ versus $f_{\mathrm{w}}$. $[\mathrm{ClPyCz}]=2.0 \mu \mathrm{M}, \lambda_{\mathrm{ex}}=325 \mathrm{~nm}, I_{0}=$ intensity at emission maximum in acetonitrile solution $\left(f_{\mathrm{w}}=0 \%\right)$.

To further study the MSP relationship in the aggregate state, PL spectra of $\mathrm{ClPyCz}$ in acetonitrile/water mixtures with different water fraction $\left(f_{\mathrm{w}}\right)$ were taken as an example. By increasing the water fraction $\left(f_{\mathrm{w}}\right)$ from 0 to $60 \%, \mathrm{ClPyCz}$ exhibited gradually red-shifted emission but decreased PL intensity (Figure 1c) due to the TICT effect triggered by the increased polarity of solvent upon water addition. At $f_{\mathrm{w}} \geq 70 \%$, a blue-shifted emission peak was observed at $385 \mathrm{~nm}$ and 
its intensity at $f_{\mathrm{w}}=99 \%$ was two times higher than that in pure acetonitrile solution (Figure 1d), demonstrating the AIE activity of ClPyCz. Results from the dynamic light scattering and powder $\mathrm{X}$-ray diffraction (XRD) also revealed the formation of nano aggregates in acetonitrile/water mixture with a high fraction (Figure S9 and S10). Generally, the internal environment of nano aggregate was less affected by solvents (ie. acetonitrile and water). ${ }^{27}$ Thus, $\mathrm{ClPyCz}$ should assume a conformation different from that in polar solution in the excited state and strong locally excitedstate emission with blue color. ${ }^{27}$ As expected, PyCz also presented AIE activity (Figures S9-S11) in the nano aggregate state because is molecular skeletons was similar to $\mathrm{ClPyCz}$. In one word, both designed compounds were AIE-active and demonstrated different molecular motion and photophysical properties from single molecule to nano aggregate.

Theoretical calculations were further carried out using time-dependent density functional theory (TD-DFT) to study the MSP relationship. In the isolated state, $\mathrm{PyCz}$ and $\mathrm{ClPyCz}$ displayed a planar conformation in $\mathrm{S}_{0}$, while they afforded a twisted molecular geometry through intramolecular rotation of $\mathrm{C}-\mathrm{N}$ bond in optimized $\mathrm{S}_{1}$ (Figure S12). Specifically, the electron clouds of the highest occupied molecular orbital and the lowest unoccupied molecular orbital are mainly localized at the carbazolyl part and the pyrimindinyl unit, respectively. Such orbital distribution was favorable for the formation of TICT state, and was also consistent with the experimental observation. Meanwhile, ONIOM method with combined quantum mechanics and molecular mechanics (QM/MM) approach was utilized to simulate the electric structures and properties of $\mathrm{PyCz}$ and $\mathrm{ClPyCz}$ in the aggregate state (Figure S13). As expected, they showed planar conformations in both ground and excited states. Besides, the lower total reorganization energy $\left(4976 \mathrm{~cm}^{-1}\right.$ for $\mathrm{PyCz}$ and $4898 \mathrm{~cm}^{-1}$ for $\left.\mathrm{ClPyCz}\right)$ in the aggregate state than that in the isolated state $\left(6814 \mathrm{~cm}^{-1}\right.$ for $\mathrm{PyCz}$ and $6894 \mathrm{~cm}^{-1}$ for $\left.\mathrm{ClPyCz}\right)$ indicated that the restriction of intramolecular motion (Figures S14 and S15). Especially, the contribution from motions of dihedral angle decreased by more than $20 \%$ in the isolated phase to less than $1 \%$ in the aggregate state due to the blockage of the rotation of $\mathrm{C}-\mathrm{N}$ bond. On the basis of above results, it was clear that the active intramolecular motion could induce the formation of flexible conformations at the single molecule level. This feature facilitated the relaxation of the excited state through nonradiative decay channel to lead to weak emission. In contrast, intramolecular motion was restricted in the aggregate state. Consequently, the conformation was less twisted and rigidified to result in strong emission.

\section{MSP study at macro aggregate level}

Luckily, centimeter-long crystalline plates were obtained and their photophysical properties were investigated. Similar to nano-aggregates, $\mathrm{ClPyCz}$ and $\mathrm{PyCz}$ also showed blue-shifted emission in the crystalline state, namely at macro aggregate level (Figure S16). These two compounds showed enhanced PL quantum yield ( $\Phi=11.6 \%$ for $\mathrm{ClPyCz} ; \Phi=11.4 \%$ for $\mathrm{PyCz}$ ) in the crystalline state than in pure acetonitrile solution ( $\Phi=2.2 \%$ for $\mathrm{ClPyCz} ; \Phi=4.3 \%$ for $\mathrm{PyCz}$ ). Thus, the photophysical properties in nano-aggregate were preserved in macro crystals due to again the restriction of intramolecular motions. Unexpectedly, the $\mathrm{ClPyCz}$ crystals showed excellent elasticity and could be bent to a cycle (bent angle $>180^{\circ}$ ) under external force (Figure 2). Such bending behavior could be repeated for many times without fatigue. Importantly, the luminescent behavior experienced no change during the bending process, indicating the robust stability of the elastic crystal. It is well known that organic crystalline materials are commonly rigid and brittle, and they 
usually crack, shatter or deform irreversibly under external stimuli. ${ }^{28-33}$ Although the elastic organic crystals have been sporadically reported, ${ }^{34-41}$ the MSP relationship of the elastic AIEgen crystals is another interesting issue without systematic research.

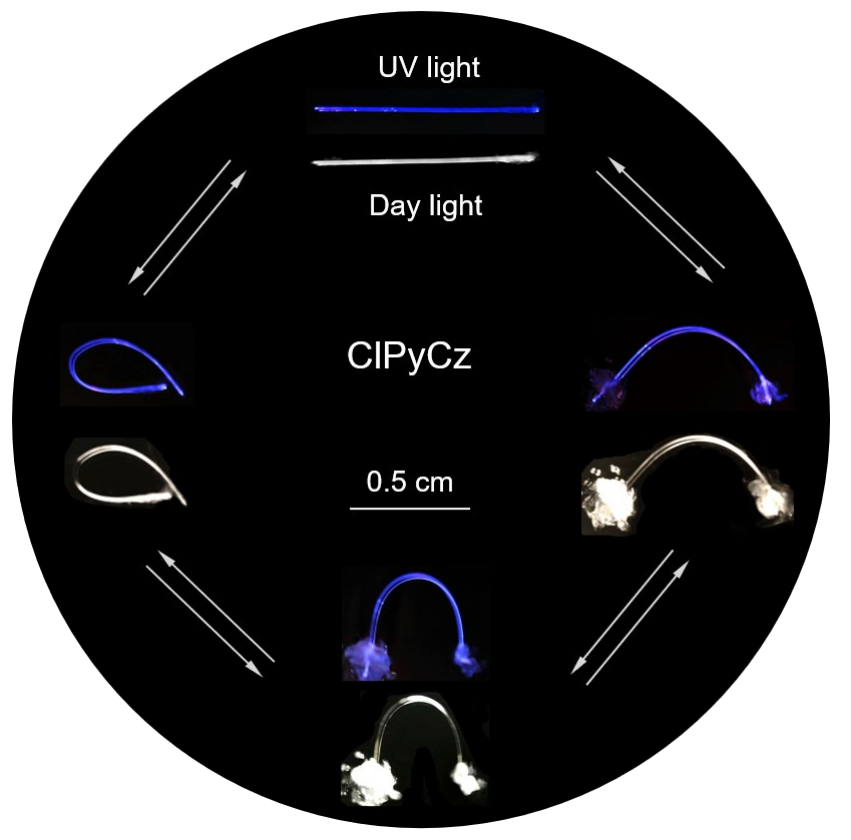

Figure 2. Photographs taken during repeated bending processes of a centimeter-long crystalline plate of $\mathrm{ClPyCz}$ under day light and UV illumination. The bent plate was fixed by high-vacuum grease at two ends.

On the contrary, $\mathrm{PhCz}$ and $\mathrm{PyCz}$ broke easily in the presence of external force even though $\mathrm{PyCz}$ had a similar molecular conformation as $\mathrm{ClPyCz}$ (Figure S17). To quantitatively ascertain the mechanical properties of the elastic ClPyCz, nanoindenter was further employed (Figure S18). Results indicated that $\mathrm{ClPyCz}$ showed an excellent performance of elasticity with a Young's modulus around $0.2 \mathrm{GPa}$, which was softer than $\mathrm{PyCz}(7.4 \mathrm{GPa})$. The obtained modulus value of ClPyCz was comparable to that of soft materials such as nylon showing a Young's modulus of 2-4 $\mathrm{GPa}^{42}$

To clarify the elastic nature, crystal structure analysis of PyCz and ClPyCz were carried out (Figures S19-S22). It was obvious that each single molecule of $\mathrm{ClPyCz}$ and $\mathrm{PyCz}$ displayed almost a planar conformation, while the model compound $\mathrm{PhCz}$ afforded a contorted molecular conformation with a dihedral angel of $60^{\circ}$ between the phenyl ring and the carbazole unit (Figure S19). On the other hand, unlike original crystals, bendable crystal usually has an elongated bendable face at the outer arc and another contracted face at the inner arc. ${ }^{32,40}$ Because of this inspiration, the bendable faces of $\mathrm{ClPyCz}$ crystal were further investigated (Figures 3 and S20). Interestingly, constant herringbone packing arrangement of $\mathrm{ClPyCz}$ molecules with multiple intermolecular interactions could be observed, as similar to many reported elastic crystals. ${ }^{29}$ These intermolecular interactions could be classified into three types, denoted as type I, II and III. They included $\mathrm{C}-\mathrm{H} \cdots \pi$ interaction (2.849-3.011 $\AA$, Type I), Cl $\cdots \pi(3.506 \AA)$ and $\pi \cdots \pi$ stacking (3.398-3.546 $\AA$ ) between two parallel molecules (Type II), as well as $\mathrm{C}-\mathrm{H} \cdots \mathrm{Cl}(3.021 \AA)$ and $\mathrm{C}-\mathrm{H} \cdots \mathrm{N}(2.811 \AA)$ interactions (Type III, Figure $3 \mathrm{c}$ ). The energy of the intermolecular interactions was calculated to evaluate their strength. 
Type II showed the largest interaction energy and was equal to $-12.28 \mathrm{kcal} / \mathrm{mol}$. While the energy of type I was $-6.93 \mathrm{kcal} / \mathrm{mol}$, that of type III was $-4.88 \mathrm{kcal} / \mathrm{mol}$ (Figure $3 \mathrm{c}$ ). According to the above data and crystal packing structure, the packing of ClPyCz molecules with type II interactions devoted to the realization of a strong intermolecular connectivity, which strongly kept the overall shape of the crystal without breaking during the bending processes. Meanwhile, the relatively weak type I and III interactions along with the herringbone arrangement allowed relative movement between closely packed molecules, which resulted in reversible compression along the interior arc and expansion along the outside arc (Figure 3d). Thus, both strong and relatively weak non-covalent intermolecular interactions were keys to synergically enable the "continuity" and "deformation" of the packing structure under external stimuli. In contrast, the brittle crystals of $\mathrm{PyCz}$ and $\mathrm{PhCz}$ showed no such packing modes and intermolecular interactions (Figure S21 and S22).

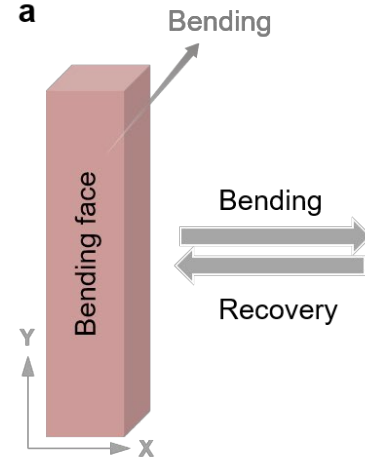

C

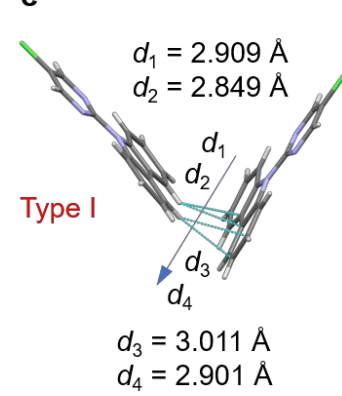

Interaction

$d_{1}-d_{4}: C-H \ldots \pi$

$-6.93 \mathrm{kcal} / \mathrm{mol}$

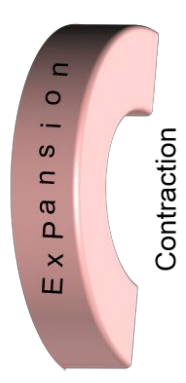

b

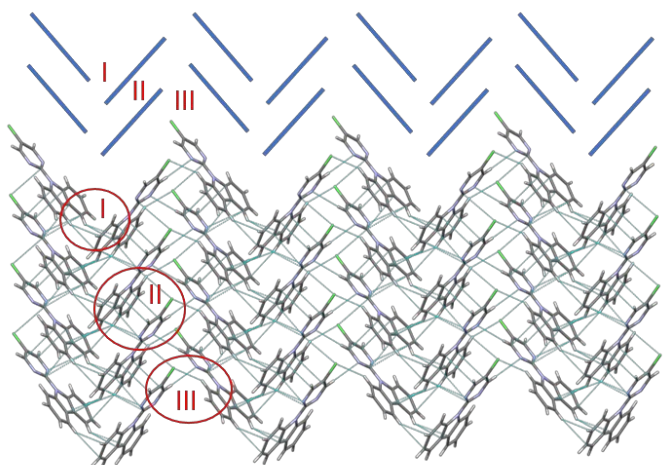

d
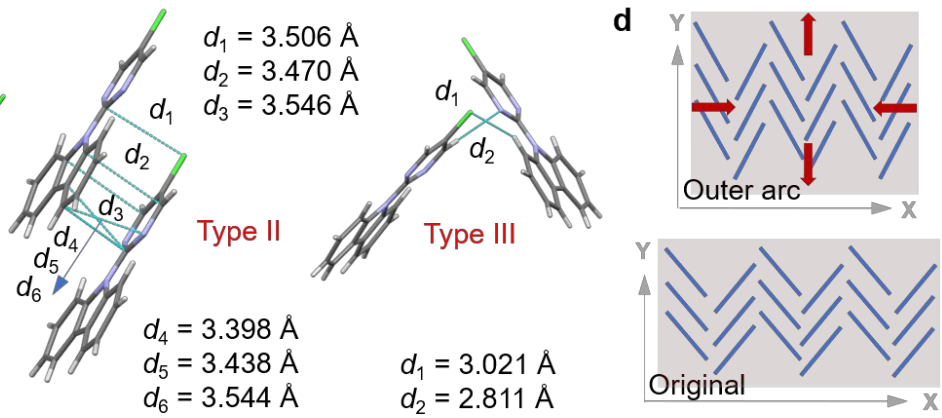

$d_{1}: \mathrm{C}-\mathrm{H} \ldots \mathrm{Cl}$

$d_{2}: \mathrm{C}-\mathrm{H} \ldots \mathrm{N}$

$-4.88 \mathrm{kcal} / \mathrm{mol}$

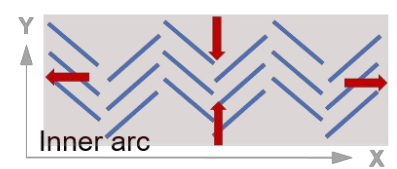

Figure 3. (a) Cartoon illustration of bending and recovery processes of an elastic organic crystal. (b) Single crystal structure of ClPyCz. (c) Packing arrangements (or $d$ spacing) and intermolecular interactions between $\mathrm{ClPyCz}$ molecules. (d) Proposed mechanism for the bending process of ClPyCz crystal.

To provide more insight on the intermolecular motions upon crystal bending, a bent crystal of ClPyCz was reexamined using single-crystal XRD. As shown in Figure S27, some diffraction spots elongated in a certain direction. Also, normal spots were observed. These diffraction spots confirmed that the periodic nature of the crystal was largely retained in the bent state. ${ }^{34}$ Besides, the elongation of spots indicated the localized the movement of the molecules away from their idealized lattice points, ie. stretching or compression of molecular packing through intermolecular motions 
(Figure 3d). However, the diffraction spots from the bent crystal could not be used to index to the original unit cell. They just demonstrated the intermolecular motions of the AIEgen, which was accorded to the above mechanistic inferences. Therefore, by tuning the molecular motions of AIEgens at the macro aggregate level, both efficient luminescence and outstanding elastic performance of crystal could be achieved.

\section{Molecular engineering of AIE-active elastic crystals}

ClPyCz served not only as a tool to monitor the molecular motion at single molecule and nanoaggregate levels, but also as a building block to construct elastic crystals. To demonstrate the general design strategy, other molecules based on the same molecular skeleton were studied. Considering the similar chemical attributes from the same group, $\mathrm{Br}$ was first selected to replace $\mathrm{Cl}$ to obtain 9(5-bromopyrimidin-2-yl)-carbazole (BrPyCz). As expected, $\mathrm{BrPyCz}$ showed solvent polaritydependent TICT emission (Figures S28-S30) and free molecular motions in the isolated state. However, upon the formation of nano aggregates in water/acetonitrile mixtures, an enhanced and blue-shifted emission was observed (Figure S31-S33). Besides, its crystal also affords blue emission with a $\Phi$ value (2.1\%) higher than that in pure acetonitrile ( $\Phi<1 \%$, Figure S34). As a result, $\mathrm{BrPyCz}$ exhibited restricted intramolecular motion and AIE property in nano aggregate and crystalline state. These observations were also verified by theoretical calculations (Figures S35-S37).

On the other hand, $\mathrm{BrPyCz}$ also presented the same herringbone packing arrangement with three types of intermolecular interactions (Figures 4, S38 and S39). As the bromine substituent was capable of forming $\mathrm{Br} \cdots \pi$ interactions. The strong intermolecular $\mathrm{Br} \cdots \pi$ and $\pi \cdots \pi$ interactions (type II), and multiple weak type I $(\mathrm{C}-\mathrm{H} \cdots \pi)$ and type III $(\mathrm{C}-\mathrm{H} \cdots \mathrm{N}$ and $\mathrm{C}-\mathrm{H} \cdots \mathrm{Br})$ intermolecular interactions were observed (Figure 4). The strongest type II interaction with an interaction energy of $-12.52 \mathrm{kcal} / \mathrm{mol}$ also contributed to the accessed intermolecular motions when bending force was applied to the crystal (Figure 4). Additionally, BrPyCz also displayed outstanding bendable behavior which could be compressed into a cycle (Figure 5). This exceptional elastic performance also matched well with the nano-indenter result with a modulus of $1.68 \mathrm{GPa}$ (Figure S40). This example again verified that manipulating motion modes by molecular aggregation or external stimuli can adjust the structures and properties of matter at different levels of structural hierarchy to finally realize AIE and elasticity simultaneously in the macro crystal. 


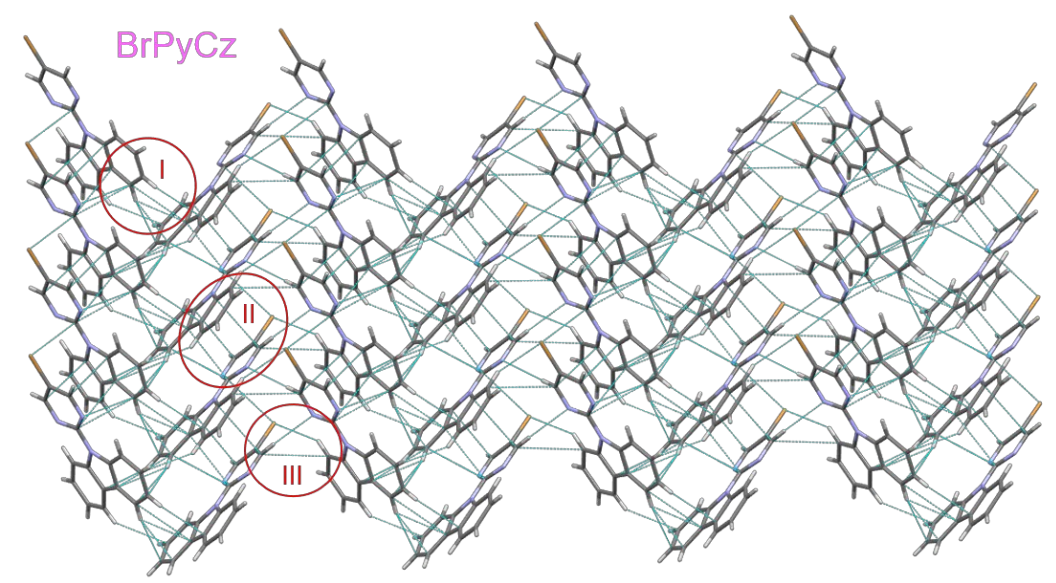

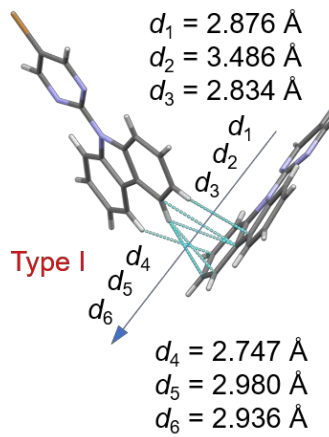

Interaction

$$
d_{1}-d_{6}: C-H-\ldots \pi
$$

$-6.55 \mathrm{kcal} / \mathrm{mol}$

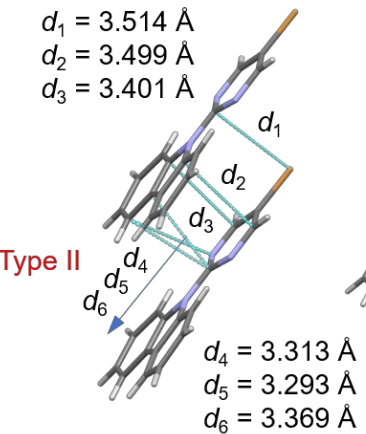

$$
d_{1}: \mathrm{Br} \cdots \pi
$$

$d_{2}-d_{6}=\pi \cdots \pi+D-A$

$-12.52 \mathrm{kcal} / \mathrm{mol}$

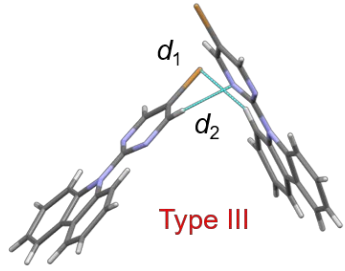

$d_{1}=3.015 \AA$

$d_{2}=2.700 \AA$

$d_{1}=\mathrm{C}-\mathrm{H} \ldots \mathrm{Br}$

$d_{2}: \mathrm{C}-\mathrm{H} \ldots \mathrm{N}$

$-5.31 \mathrm{kcal} / \mathrm{mol}$

Figure 4. Upper panel: single crystal structure of $\mathrm{BrPyCz}$. Lower panel: packing arrangements (or $d$-spacing) and intermolecular interactions between $\mathrm{BrPyCz}$ molecules.

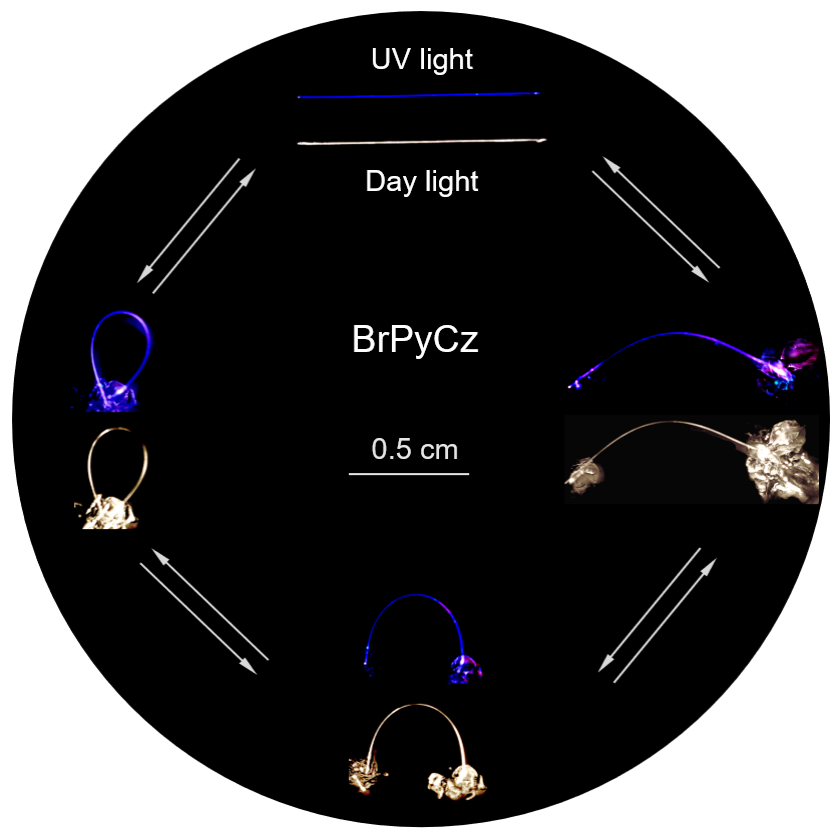

Figure 5. Photographs taken during repeated bending processes of a centimeter-long crystalline plate of $\mathrm{BrPyCz}$ under day light and UV illumination. The bent plate was fixed by high-vacuum grease at two ends. 
In addition, cyano group, which served as a pseudo halogen, was also chosen to replace the halogen atoms $(\mathrm{Cl}$ or $\mathrm{Br})$ to construct 9-(5-cyanopyrimidin-2-yl)-carbazole $(\mathrm{CyPyCz})$. Owing to the $\pi$ conjugation and rigid structure of the cyano group, $\mathrm{CyPyCz}$ showed more redder and efficient emission than $\mathrm{ClPyCz}$ and $\mathrm{BrPyCz}$. Its photophysical properties (Figure S41-S47), theoretical calculations (Figures 6 and S48-50), crystal structure (Figures 6 and S51), and mechanical property (Figure S53) were systematically studied. Results showed that CyPyCz demonstrated TICT effect in solution and AIE property in nano aggregate and macro crystal. Particularly, its efficient blue emission at around $403 \mathrm{~nm}$ with a $\Phi$ of $15.4 \%$ could be observed from macro crystal (Figure S47), which was consistent with our molecular design. More importantly, $\mathrm{CyPyCz}$ also afforded three types of packing arrangement with strong and weak intermolecular interactions as indicated by both interaction distances and theoretical calculation (Figures 6, S51 and S52). As a result, the macro crystal of $\mathrm{CyPyCz}$ with a length $>1 \mathrm{~cm}$ showed good elastic behavior and could be bent freely (Figures 7 and 53). Clearly, the molecular engineering for achieving AIE-active elastic organic crystal by manipulating the intra/intermolecular motions was realized and demonstrated.

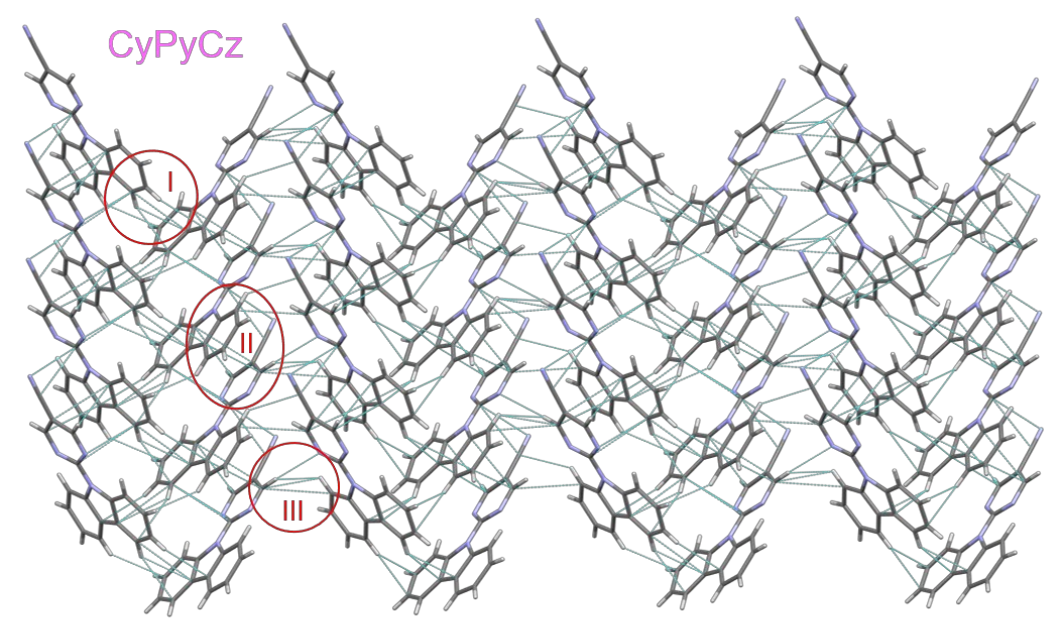

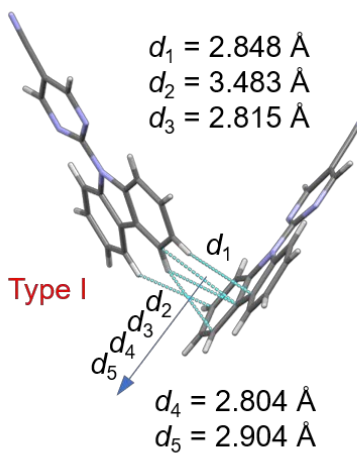

Interaction

$$
\begin{gathered}
d_{1}-d_{5}: \mathrm{C}-\mathrm{H} \cdots \pi \\
-6.93 \mathrm{kcal} / \mathrm{mol}
\end{gathered}
$$

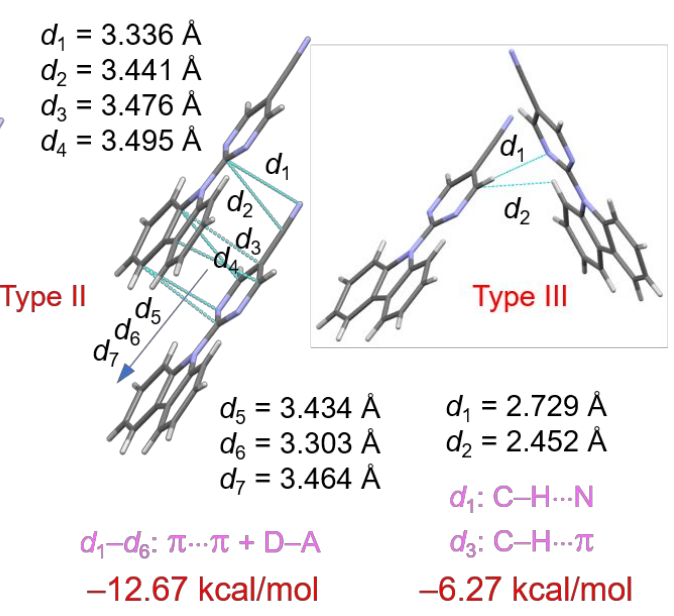

Figure 6. Upper panel: single crystal structure of CyPyCz. Lower panel: packing arrangements (or $d$-spacing) and intermolecular interactions between CyPyCz molecules. 


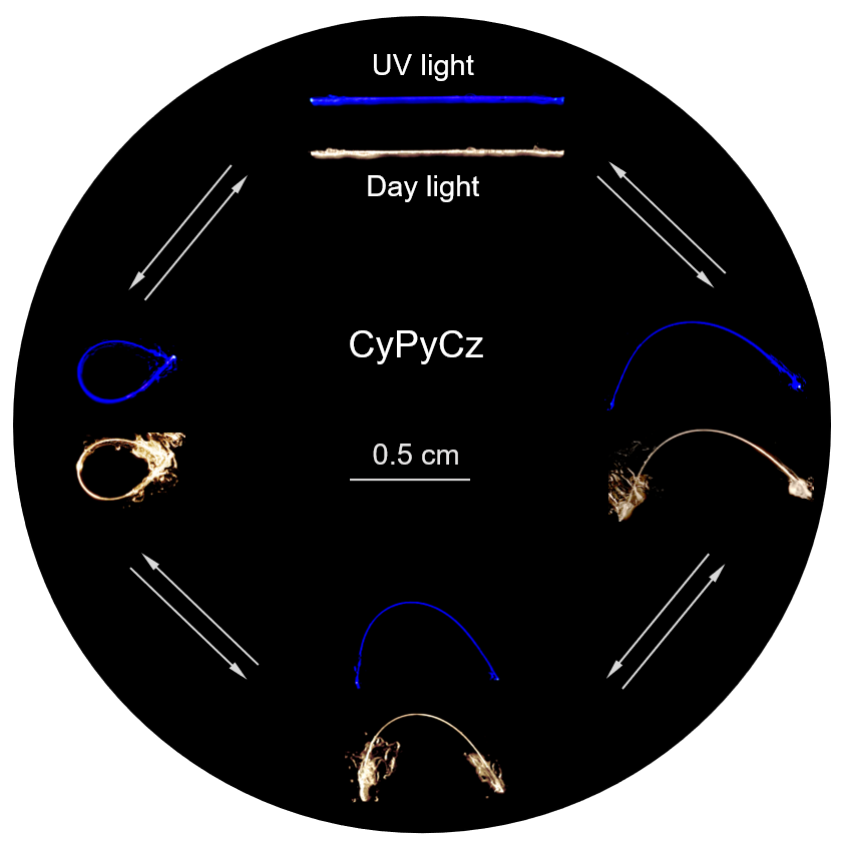

Figure 7. Photographs taken during repeated bending processes of a centimeter-long crystalline plate of $\mathrm{CyPyCz}$ under day light and UV illumination. The bent plate was fixed by high-vacuum grease at two ends.

\section{Conclusion}

In this study, we demonstrated that molecular motions of AIEgens could affect their structures and properties. By melding electron-donating luminophores with electron-deficient molecular rotors, the obtained AIEgens not only enabled us to exploration of motion-structure-property relationship at different levels of structural hierarchy from molecule to macro aggregate, but also contributed to the realization of AIE-active elastic organic crystals. Importantly, molecular engineering for AIEactive elastic organic crystals could be generally demonstrated by designing molecules with a same molecular skeleton. Thus, this study had witnessed that AIE and elasticity could be simultaneously obtained within one molecule. In light of both the experimental and theoretical results, we believed that intra/intermolecular motions indeed could devote to structural tuning at each level of hierarchy, and further affecting the inherent photophysical/mechanical behaviors. Through manipulating the MSP relationship, aggregates or molecule with remarkable properties such as mechanical, magnetic and electronic properties might be generated. It was anticipated that the present study could exert impact on the existing AIE research, and provide new prospects for aggregate science. 


\section{References}

1. Grabowski, Z. R.; Rotkiewicz, K.; Rettig, W., Structural changes accompanying intramolecular electron transfer: focus on twisted intramolecular charge-transfer states and structures. Chem. Rev. 2003, 103 (10), 3899-4032.

2. Serrano-Andres, L.; Merchan, M.; Roos, B. O.; Lindh, R., Theoretical Study of the Internal Charge Transfer in Aminobenzonitriles. J. Am. Chem. Soc. 1995, 117 (11), 3189-3204.

3. Horie, M.; Wang, C.-H., Stimuli-responsive dynamic pseudorotaxane crystals. Mater. Chem. Front. 2019, 3 (11), 2258-2269.

4. Zhang, J.-P.; Zhou, H.-L.; Zhou, D.-D.; Liao, P.-Q.; Chen, X.-M., Controlling flexibility of metalorganic frameworks. Natl. Sci. Rev. 2018, 5 (6), 907-919.

5. Wang, H.; Chen, P.; Wu, Z.; Zhao, J.; Sun, J.; Lu, R., Bending, Curling, Rolling, and Salient Behavior of Molecular Crystals Driven by [2+2] Cycloaddition of a styrylbenzoxazole derivative. Angew. Chem. Int. Ed. 2017, 56 (32), 9463-9467.

6. Paeng, K.; Swallen, S. F.; Ediger, M. D., Direct measurement of molecular motion in freestanding polystyrene thin films. J. Am. Chem. Soc. 2011, 133 (22), 8444-8447.

7. Comotti, A.; Bracco, S.; Yamamoto, A.; Beretta, M.; Hirukawa, T.; Tohnai, N.; Miyata, M.; Sozzani, P., Engineering Switchable rotors in molecular crystals with open porosity. J. Am. Chem. Soc. 2014, 136 (2), 618-621.

8. Zhang, J.; Zhang, H.; Liu, J.; Lam, J. W. Y.; Tang, B. Z., Visualizing changes of molecular conformation in the solid-state by a common structural determination technique: single crystal X-ray diffraction. Mater. Chem. Front. 2021, 5 (1), 341-346.

9. Zhang, H.; Du, L.; Wang, L.; Liu, J.; Wan, Q.; Kwok, R. T. K.; Lam, J. W. Y.; Phillips, D. L.; Tang, B. Z., Visualization and manipulation of molecular motion in the solid state through photoinduced clusteroluminescence. J. Phys. Chem. Lett. 2019, 10 (22), 7077-7085.

10. Aguilar-Granda, A.; García-González, M. C.; Pérez-Estrada, S.; Kozina, A.; Rodríguez-Molina, B., Nanoscale organization and solid-state dynamics of carbazole- $[\pi]$-carbazole rotors edged with aliphatic chains. J. Phys. Chem. C 2018, 122 (47), 27093-27099.

11. Alam, P.; Leung, N. L. C.; Cheng, Y.; Zhang, H.; Liu, J.; Wu, W.; Kwok, R. T. K.; Lam, J. W. Y.; Sung, H. H. Y.; Williams, I. D.; Tang, B. Z., Spontaneous and fast molecular motion at room temperature in the solid state. Angew. Chem. Int. Ed. 2019, 58 (14), 4536-4540.

12. Luo, J.; Xie, Z.; Lam, J. W. Y.; Cheng, L.; Chen, H.; Qiu, C.; Kwok, H. S.; Zhan, X.; Liu, Y.; Zhu, D.; Tang, B. Z., Aggregation-induced emission of 1-methyl-1,2,3,4,5-pentaphenylsilole. Chem. Commun. 2001, (18), 1740-1741.

13. Zhao, Z.; Zhang, H.; Lam, J. W. Y.; Tang, B. Z., Aggregation-induced emission: new vistas at the aggregate level. Angew. Chem. Int. Ed. 2020, 59 (25), 9888-9907.

14. Tu, Y.; Zhao, Z.; Lam, J. W. Y.; Tang, B. Z., Aggregate Science: Much to Explore in the Meso World. Matter 2021, 4 (2), 338-349.

15. Hu, F.; Mao, D.; Kenry; Cai, X.; Wu, W.; Kong, D.; Liu, B., A light-up probe with aggregationinduced emission for real-time bio-orthogonal tumor labeling and image-guided photodynamic therapy. Angew. Chem. Int. Ed. 2018, 57 (32), 10182-10186.

16. Zhang, H.; Zhao, Z.; Turley, A. T.; Wang, L.; McGonigal, P. R.; Tu, Y.; Li, Y.; Wang, Z.; Kwok, R. T. K.; Lam, J. W. Y.; Tang, B. Z., Aggregate science: from structures to properties. Adv. Mater. 2020, 32 (36), 2001457. 
17. Zhang, R.; Duan, Y.; Liu, B., Recent advances of AIE dots in NIR imaging and phototherapy. Nanoscale 2019, 11 (41), 19241-19250.

18. Feng, G.; Zhang, G.-Q.; Ding, D., Design of superior phototheranostic agents guided by Jablonski diagrams. Chem. Soc. Rev. 2020, 49 (22), 8179-8234.

19. Kong, Y.-J.; Yan, Z.-P.; Li, S.; Su, H.-F.; Li, K.; Zheng, Y.-X.; Zang, S.-Q., Photoresponsive Propeller-like Chiral AIE Copper(I) Clusters. Angew. Chem. Int. Ed. 2020, 59 (13), 5336-5340.

20. Han, X.; Ge, F.; Xu, J.; Bu, X.-H., Aggregation-induced emission materials for nonlinear optics. Aggregate 2021, e28.

21. Lou, X.-Y.; Yang, Y.-W., Aggregation-induced emission systems involving supramolecular assembly. Aggregate 2020, 1, 19-30.

22. Hong, Y.; Lam, J. W. Y.; Tang, B. Z., Aggregation-induced emission: phenomenon, mechanism and applications. Chem.Commun. 2009, 4332-4353.

23. Hong, Y.; Lam, J. W. Y.; Tang, B. Z., Aggregation-induced emission. Chem. Soc. Rev. 2011, 40 (11), 5361-5388.

24. Wu, W.; Liu, B., Aggregation-induced emission: challenges and opportunities. Natl. Sci. Rev. 2020.

25. Zhang, J.; Z. H., Lam J. W. Y., Tang, B. Z., Restriction of intramolecular motion (RIM): investigating aie mechanism from experimental and theoretical studies. Chem. Res. Chin. Univ. 2021, 37 (1), 1-15.

26. Meng, L.; Ma, X.; Jiang, S.; Zhang, S.; Wu, Z.; Xu, B.; Lei, Z.; Liu, L.; Tian, W., Twisted intramolecular charge transfer-aggregation-induced emission fluorogen with polymer encapsulationenhanced near-infrared emission for bioimaging. CCS Chemistry 2021, 3 (3), 2084-2094.

27. Hu, R.; Lager, E.; Aguilar-Aguilar, A.; Liu, J.; Lam, J. W. Y.; Sung, H. H. Y.; Williams, I. D.; Zhong, Y.; Wong, K. S.; Peña-Cabrera, E.; Tang, B. Z., Twisted intramolecular charge transfer and aggregationinduced emission of BODIPY derivatives. J. Phys. Chem. C 2009, 113 (36), 15845-15853.

28. Liu, H.; Lu, Z.; Zhang, Z.; Wang, Y.; Zhang, H., Highly elastic organic crystals for flexible optical waveguides. Angew. Chem. Int. Ed. 2018, 57 (28), 8448-8452.

29. Huang, R.; Wang, C.; Wang, Y.; Zhang, H., Elastic self-doping organic single crystals exhibiting flexible optical waveguide and amplified spontaneous emission. Adv. Mater. 2018, 30 (21), 1800814.

30. Liu, B.; Lu, Z.; Tang, B.; Liu, H.; Liu, H.; Zhang, Z.; Ye, K.; Zhang, H., Self-waveguide singlebenzene organic crystal with ultralow-temperature elasticity as a potential flexible material. Angew. Chem. Int. Ed. 2020, 59 (51), 23117-23121.

31. Liu, H.; Ye, K.; Zhang, Z.; Zhang, H., An organic crystal with high elasticity at an ultra-low temperature (77 k) and shapeability at high temperatures. Angew. Chem. Int. Ed. 2019, 58 (52), 1908119086.

32. Liu, H.; Lu, Z.; Tang, B.; Qu, C.; Zhang, Z.; Zhang, H., A flexible organic single crystal with plastictwisting and elastic-bending capabilities and polarization-rotation function. Angew. Chem. Int. Ed. 2020, 59 (31), 12944-12950.

33. Lu, Z.; Zhang, Y.; Liu, H.; Ye, K.; Liu, W.; Zhang, H., Optical waveguiding organic single crystals exhibiting physical and chemical bending features. Angew. Chem. Int. Ed. 2020, 59 (11), 4299-4303.

34. Ghosh, S.; Reddy, C. M., Elastic and bendable caffeine cocrystals: implications for the design of flexible organic materials. Angew. Chem. Int. Ed. 2012, 51 (41), 10319-10323.

35. Ghosh, S.; Mishra, M. K.; Kadambi, S. B.; Ramamurty, U.; Desiraju, G. R., Designing elastic organic crystals: highly flexible polyhalogenated n-benzylideneanilines. Angew. Chem. Int. Ed. 2015, 54 (9), 2674-2678. 
36. Krishna, G. R.; Devarapalli, R.; Lal, G.; Reddy, C. M., Mechanically flexible organic crystals achieved by introducing weak interactions in structure: supramolecular shape synthons. J. Am. Chem. Soc. 2016, 138 (41), 13561-13567.

37. Mondal, A.; Bhattacharya, B.; Das, S.; Bhunia, S.; Chowdhury, R.; Dey, S.; Reddy, C. M., Metallike ductility in organic plastic crystals: role of molecular shape and dihydrogen bonding interactions in aminoboranes. Angew. Chem. Int. Ed. 2020, 59 (27), 10971-10980.

38. Hayashi, S.; Koizumi, T., Elastic organic crystals of a fluorescent $\pi$-conjugated molecule. Angew. Chem. Int. Ed. 2016, 55 (8), 2701-2704.

39. Hayashi, S.; Yamamoto, S.-y.; Takeuchi, D.; Ie, Y.; Takagi, K., Creating elastic organic crystals of $\pi$-conjugated molecules with bending mechanofluorochromism and flexible optical waveguide. Angew. Chem. Int. Ed. 2018, 57 (52), 17002-17008.

40. Worthy, A.; Grosjean, A.; Pfrunder, M. C.; Xu, Y.; Yan, C.; Edwards, G.; Clegg, J. K.; McMurtrie, J. C., Atomic resolution of structural changes in elastic crystals of copper(II) acetylacetonate. Nat. Chem. 2018, 10 (1), 65-69.

41. Saha, S.; Mishra, M. K.; Reddy, C. M.; Desiraju, G. R., From molecules to interactions to crystal engineering: mechanical properties of organic solids. Acc. Chem. Res. 2018, 51 (11), 2957-2967.

42. Somiya, S., Handbook of advanced ceramics: materials, applications, processing, and properties. Academic press: 2013. 
Structural Hierarchy and Behavioral Diversity (from Molecule to Aggregate)

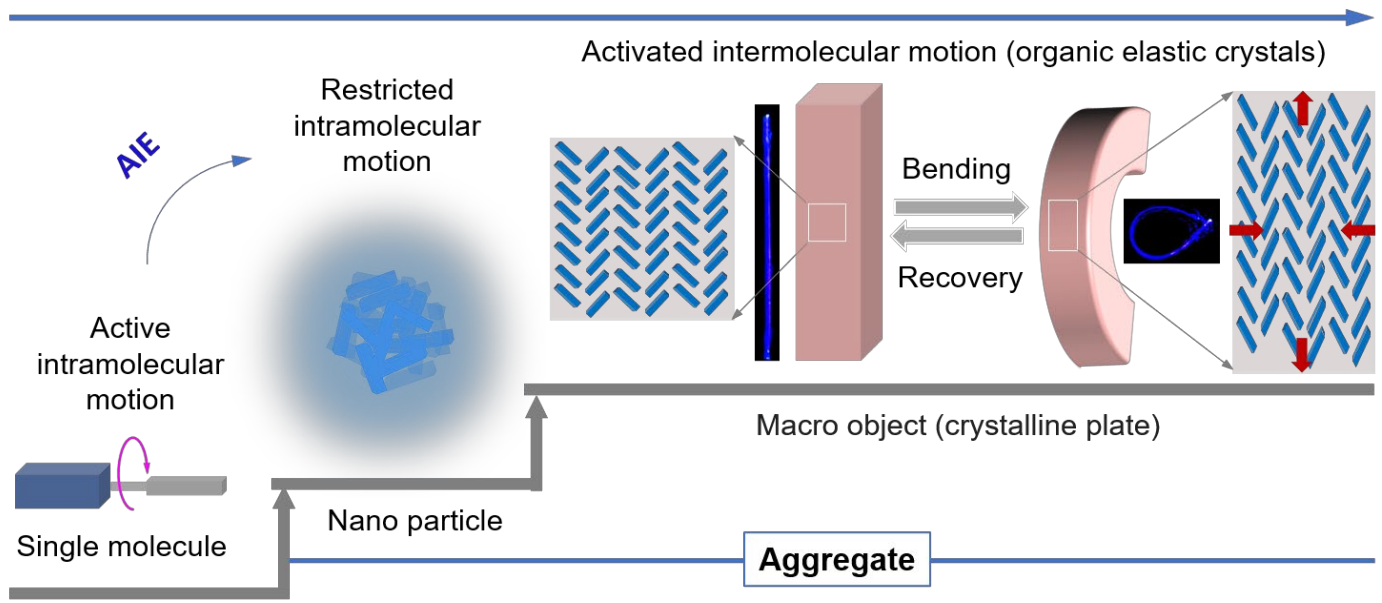

